\title{
ON THE THEORY OF THIN ELASTIC SHELLS*
}

\author{
BY \\ P. M. NAGHDI \\ University of Michigan
}

1. Introduction. The linear theory of thin elastic shells has received attention by numerous authors who have employed a variety of approximations in their work. Inasmuch as there is no difficulty in obtaining the stress differential equations of equilibrium and expressions for the components of strain, consistent with the assumptions for displacements, the works of these authors differ from one another essentially in the formulation of appropriate stress strain relations. With a few exceptions, the approximations introduced have been within the framework of the classical shell theory where, in addition to the smallness of (a) the thickness $h$ in comparison with the least radius of curvature of the middle surface $R$, i.e., $h / R \ll 1$, (b) strains and displacements so that the quantities of second and higher order terms are neglected in comparison with the first order terms, it is also assumed that (c) the component of stress normal to the middle surface is small compared with other components of stress and it may, therefore, be neglected in the stress strain relations, and (d) plane cross sections normal to the undeformed middle surface remain normal to the deformed middle surface and suffer no extension. The last assumption implies neglect of the transverse shear deformation.

Recent and notable contributions, where the effects of both transverse normal stress and shear deformation have been accounted for, are by Hildebrand, E. Reissner and Thomas [1], by Green and Zerna [2], and by E. Reissner [3] where references are made to previous works. From a practical point of view, reference [3], which is restricted to axisymmetric deformation of shells of revolution with elastically preferred directions along the normals to the middle surface, i.e., sandwich shells, contains results which represent some simplifications as compared with those given in [1] and [2].

The present paper is concerned with the formulation of suitable stress strain relations and the appropriate boundary conditions in the theory of small deformation of thin elastic isotropic shells of uniform thickness. The results, which include the effects of transverse normal stress, transverse shear deformation, as well as rotary inertia (discussed separately in Sec. 4), are deduced by application of a recent variational theorem due to E. Reissner [4]. Since for the most part the underlying derivation for the stress strain relations is similar to that of reference [3], details of computation are omitted; it is felt, however, that the presentation of the final results will serve a useful purpose.

2. The coordinate system, notation, and preliminaries. Let $\xi_{1}$ and $\xi_{2}$ be the coordinates of a point on the middle surface of the shell and $\zeta$ be the distance measured along the outward normal to the middle surface. Further, let $\mathbf{n}$ be the unit normal vector at a point of the middle surface and $t_{1}$ and $t_{2}\left(t_{1}, t_{2}\right.$ and $n$ form a right-handed system) be the unit tangent vectors to the $\xi_{1}$ - and $\xi_{2}$-curves, respectively. Then the coordinate curves $\xi_{1}$ and $\xi_{2}$ as lines of curvature (on the middle surface, $\zeta=0$ ) together

${ }^{*}$ Received December 5, 1955. The results presented in this paper were obtained in the course of research sponsored by the Office of Naval Research under Contract Nonr-1224 (01), Project NR-064408, with the University of Michigan. 
with $\zeta$, specify the position of a point in space. The square of linear element of this triply orthogonal coordinate system may be shown to be

$$
d s^{2}=\alpha_{1}^{2}\left(1+\frac{\zeta}{R_{1}}\right)^{2} d \xi_{1}^{2}+\alpha_{2}^{2}\left(1+\frac{\zeta}{R_{2}}\right)^{2} d \xi_{2}+d \zeta^{2},
$$

where $R_{1}$ and $R_{2}$ are the principal radii of curvature of the middle surface. We note here for future reference the formulas

$$
\begin{aligned}
& \frac{\partial}{\partial \xi_{2}}\left[\alpha_{1}\left(1+\frac{\zeta}{R_{1}}\right)\right]=\left(1+\frac{\zeta}{R_{2}}\right) \frac{\partial \alpha_{1}}{\partial \xi_{2}} \\
& \frac{\partial}{\partial \xi_{1}}\left[\alpha_{2}\left(1+\frac{\zeta}{R_{2}}\right)\right]=\left(1+\frac{\zeta}{R_{1}}\right) \frac{\partial \alpha_{2}}{\partial \xi_{1}}
\end{aligned}
$$

which may be deduced from the well-known Mainardi-Codazzi relations.

The displacement vector $U$ of a point in space will be conveniently written as

$$
\mathbf{U}=U_{1} \mathbf{t}_{1}+U_{2} \mathbf{t}_{2}+W \mathbf{n}
$$

and the six components of strain in the curvilinear coordinate system $\xi_{1}, \xi_{2}, \zeta$, with the aid of (2.2), (see Ref. [5], p. 51), are:

$$
\begin{aligned}
\epsilon_{1} & =\left[\alpha_{1}\left(1+\frac{\zeta}{R_{1}}\right)\right]^{-1}\left\{\frac{\partial U_{1}}{\partial \xi_{1}}+\frac{U_{2}}{\alpha_{2}} \frac{\partial \alpha_{1}}{\partial \xi_{2}}+\alpha_{1} \frac{W}{R_{1}}\right\}, \\
\epsilon_{2} & =\left[\alpha_{2}\left(1+\frac{\zeta}{R_{2}}\right)\right]^{-1}\left\{\frac{\partial U_{2}}{\partial \xi_{2}}+\frac{U_{1}}{\alpha_{1}} \frac{\partial \alpha_{2}}{\partial \xi_{1}}+\alpha_{2} \frac{W}{R_{2}}\right\}, \\
\epsilon_{\zeta} & =\frac{\partial W}{\partial \zeta}, \\
\gamma_{12} & =\left[\frac{\alpha_{2}}{\alpha_{1}}\left(1+\frac{\zeta}{R_{2}}\right)\left(1+\frac{\zeta}{R_{1}}\right)^{-1}\right] \frac{\partial}{\partial \xi_{1}}\left\{\frac{U_{2}}{\alpha_{2}}\left(1+\frac{\zeta}{R_{2}}\right)^{-1}\right\} \\
\gamma_{15} & \left.\left.=\left[\alpha_{1}\left(1+\frac{\zeta}{\alpha_{2}}\right)\right]^{-1} \frac{\partial W}{\partial \xi_{1}}+\left(1+\frac{\zeta}{R_{1}}\right)\left(1+\frac{\zeta}{R_{2}}\right)^{-1}\right] \frac{\partial}{\partial \xi_{2}}\left\{\frac{U_{1}}{\alpha_{1}}\left(1+\frac{\zeta}{R_{1}}\right)_{1}\right)^{-1}\right\}, \\
\gamma_{2 \zeta} & =\left[U_{1}\left(1+\frac{\zeta}{R_{1}}\right)^{-1}\right\},
\end{aligned}
$$

The normal components of stress will be denoted by $\sigma_{1}, \sigma_{2}$, and $\sigma_{\xi}$, while the shearing stresses will be designated by $\tau_{12}, \tau_{15}$ and $\tau_{2 \zeta}$. The stress resultants $N_{1}, N_{2}, N_{12}$ and $N_{21}$; the stress couples $M_{1}, M_{2}, M_{12}$ and $M_{21}$; and the shear resultants $V_{1}$ and $V_{2}$ are defind in the conventional manner by the expressions of the type:

$$
\begin{aligned}
& \left\{\begin{array}{l}
N_{1} \\
M_{1} \\
V_{1}
\end{array}\right\}=\int_{-h / 2}^{+h / 2}\left\{\begin{array}{l}
\sigma_{1} \\
\zeta \sigma_{1} \\
\tau_{1 \zeta}
\end{array}\right\}\left(1+\frac{\zeta}{R_{2}}\right) d \zeta, \\
& \left\{\begin{array}{l}
N_{21} \\
M_{21}
\end{array}\right\}=\int_{-h / 2}^{+h / 2}\left\{\begin{array}{l}
\tau_{12} \\
\zeta \tau_{12}
\end{array}\right\}\left(1+\frac{\zeta}{R_{1}}\right) d \zeta, \quad \text { etc. }
\end{aligned}
$$


and these are related to the stress resultant and stress couple vectors by

$$
\begin{array}{ll}
\mathbf{N}_{1}=N_{1} \mathbf{t}_{1}+N_{12} \mathbf{t}_{2}+V_{1} \mathbf{n}, & \mathbf{N}_{2}=N_{21} \mathbf{t}_{1}+N_{2} \mathbf{t}_{2}+V_{2} \mathbf{n}, \\
\mathbf{M}_{1}=M_{12} \mathbf{t}_{1}-M_{1} \mathbf{t}_{2}, & \mathbf{M}_{2}=M_{2} \mathbf{t}_{1}-M_{21} \mathbf{t}_{2} .
\end{array}
$$

Before obtaining the appropriate stress strain relations, it is necessary to introduce approximations for the displacements and stresses. For this purpose, we assume for the displacements the form

$$
U_{1}=u_{1}+\zeta \beta_{1}, \quad U_{2}=u_{2}+\zeta \beta_{2}, \quad W=w+\zeta w^{\prime}+\frac{1}{2} \zeta^{2} w^{\prime \prime},
$$

where $u_{1}, u_{2}$, and $w$ are the components of displacements at the middle surface; $\beta_{1}$ and $\beta_{2}$ are the changes of slope of the normal to the middle surface; and $w^{\prime}$ and $w^{\prime \prime}$ are the contributions to the transverse normal strain.

Introduction of (2.7) into (2.4) leads to the following expressions for the components of strain:

$$
\begin{aligned}
\left(1+\frac{\zeta}{R_{1}}\right) \epsilon_{1} & =\epsilon_{1}^{0}+\zeta \kappa_{1}^{*}+\frac{1}{2} \zeta^{2} \frac{w^{\prime \prime}}{R_{1}} \\
\left(1+\frac{\zeta}{R_{2}}\right) \epsilon_{2} & =\epsilon_{2}^{0}+\zeta \kappa_{2}^{*}+\frac{1}{2} \zeta^{2} \frac{w^{\prime \prime}}{R_{2}} \\
\epsilon_{\zeta} & =w^{\prime}+\zeta w^{\prime \prime} \\
\left(1+\frac{\zeta}{R_{1}}\right)\left(1+\frac{\zeta}{R_{2}}\right) \gamma_{12} & =\left(1+\frac{\zeta}{R_{2}}\right)\left(\gamma_{1}^{0}+\zeta \delta_{1}\right)+\left(1+\frac{\zeta}{R_{1}}\right)\left(\gamma_{2}^{0}+\zeta \delta_{2}\right) \\
\left(1+\frac{\zeta}{R_{1}}\right) \gamma_{1 \zeta} & =\gamma_{1 \zeta}^{0}+\frac{\zeta}{\alpha_{1}}\left[\frac{\partial w^{\prime}}{\partial \xi_{1}}+\frac{\zeta}{2} \frac{\partial w^{\prime \prime}}{\partial \xi_{1}}\right] \\
\left(1+\frac{\zeta}{R_{2}}\right) \gamma_{2 \zeta} & =\gamma_{2 \zeta}^{0}+\frac{\zeta}{\alpha_{2}}\left[\frac{\partial w^{\prime}}{\partial \xi_{2}}+\frac{\zeta}{2} \frac{\partial w^{\prime \prime}}{\partial \xi_{2}}\right]
\end{aligned}
$$

where

$$
\begin{array}{rlrl}
\epsilon_{1}^{0} & =\frac{1}{\alpha_{1}}\left(\frac{\partial u_{1}}{\partial \xi_{1}}+\frac{u_{2}}{\alpha_{2}} \frac{\partial \alpha_{1}}{\partial \xi_{2}}\right)+\frac{w}{R_{1}}, & \epsilon_{2}^{0}=\frac{1}{\alpha_{2}}\left(\frac{\partial u_{2}}{\partial \xi_{2}}+\frac{u_{1}}{\alpha_{1}} \frac{\partial \alpha_{2}}{\partial \xi_{1}}\right)+\frac{w}{R_{2}} \\
\gamma_{1}^{0}=\frac{1}{\alpha_{1}}\left(\frac{\partial u_{2}}{\partial \xi_{1}}-\frac{u_{1}}{\alpha_{2}} \frac{\partial \alpha_{1}}{\partial \xi_{2}}\right), & \gamma_{2}^{0}=\frac{1}{\alpha_{2}}\left(\frac{\partial u_{1}}{\partial \xi_{2}}-\frac{u_{2}}{\alpha_{1}} \frac{\partial \alpha_{2}}{\partial \xi_{1}}\right), \\
\gamma_{15}^{0}=\frac{1}{\alpha_{1}} \frac{\partial w}{\partial \xi_{1}}-\frac{u_{1}}{R_{1}}+\beta_{1}, & \gamma_{25}^{0}=\frac{1}{\alpha_{2}} \frac{\partial w}{\partial \xi_{2}}-\frac{u_{2}}{R_{2}}+\beta_{2},
\end{array}
$$

and

$$
\begin{array}{ll}
\kappa_{1}^{*}=\kappa_{1}+\frac{w^{\prime}}{R_{1}}, & \kappa_{2}^{*}=\kappa_{2}+\frac{w^{\prime}}{R_{2}}, \\
\kappa_{1}=\frac{1}{\alpha_{1}}\left(\frac{\partial \beta_{1}}{\partial \xi_{1}}+\frac{\beta_{2}}{\alpha_{2}} \frac{\partial \alpha_{1}}{\partial \xi_{2}}\right), & \kappa_{2}=\frac{1}{\alpha_{2}}\left(\frac{\partial \beta_{2}}{\partial \xi_{2}}+\frac{\beta_{1}}{\alpha_{1}} \frac{\partial \alpha_{2}}{\partial \xi_{1}}\right), \\
\delta_{1}=\frac{1}{\alpha_{1}}\left(\frac{\partial \beta_{2}}{\partial \xi_{1}}-\frac{\beta_{1}}{\alpha_{2}} \frac{\partial \alpha_{1}}{\partial \xi_{2}}\right), & \delta_{2}=\frac{1}{\alpha_{2}}\left(\frac{\partial \beta_{1}}{\partial \xi_{2}}-\frac{\beta_{2}}{\alpha_{1}} \frac{\partial \alpha_{2}}{\partial \xi_{1}}\right) .
\end{array}
$$


Guided by the stress differential equations of equilibrium and the expression of the type (2.5), we write for the components of stress the following expressions which are consistent with the approximation (2.7):

$$
\begin{aligned}
&\left(1+\frac{\zeta}{R_{2}}\right) \sigma_{1}=\frac{N_{1}}{h}+\frac{M_{1}}{h^{2} / 6} \frac{\zeta}{h / 2}, \\
&\left(1+\frac{\zeta}{R_{1}}\right) \sigma_{2}= \frac{N_{2}}{h}+\frac{M_{2}}{h^{2} / 6} \frac{\zeta}{h / 2}, \\
&\left(1+\frac{\zeta}{R_{2}}\right) \tau_{12}= \frac{N_{12}}{h}+\frac{M_{12}}{h^{2} / 6} \frac{\zeta}{h / 2}, \\
&\left(1+\frac{\zeta}{R_{2}}\right) \tau_{1 \zeta}= \frac{3}{2} \frac{V_{1}}{h}\left[1-\left(\frac{\zeta}{h / 2}\right)^{2}\right]-\frac{1}{4}\left\{p_{1}^{+} H_{1}^{+}\left[1-2\left(\frac{\zeta}{h / 2}\right)-3\left(\frac{\zeta}{h / 2}\right)^{2}\right]\right. \\
&\left.+p_{1}^{-} H_{1}^{-}\left[1+2\left(\frac{\zeta}{h / 2}\right)-3\left(\frac{\zeta}{h / 2}\right)^{2}\right]\right\}, \\
&\left(1+\frac{\zeta}{R_{1}}\right) \tau_{2 \zeta}= \frac{3}{2} \frac{V_{2}}{h}\left[1-\left(\frac{\zeta}{h / 2}\right)^{2}\right]-\frac{1}{4}\left\{p_{2}^{+} H_{2}^{+}\left[1-2\left(\frac{\zeta}{h / 2}\right)-3\left(\frac{\zeta}{h / 2}\right)^{2}\right]\right. \\
&\left(1+\frac{\zeta}{R_{1}}\right)\left(1+\frac{\zeta}{R_{2}}\right) \sigma_{\zeta}= c\left\{\left[\frac{3}{2} \frac{S}{h}+\frac{T}{4 h}\left(\frac{\zeta}{h / 2}\right)\right]\left[1-3\left(\frac{\zeta}{h / 2}\right)^{2}\right]\right\}, \\
&\left.\left.+\frac{\zeta}{h / 2}\right)^{2}\right]+\frac{1}{2} q^{+} H^{+}\left[1+\frac{3}{2}\left(\frac{\zeta}{h / 2}\right)-\frac{1}{2}\left(\frac{\zeta}{h / 2}\right)^{-}\right] \\
&\left.-\left[1-\frac{3}{2}\left(\frac{\zeta}{h / 2}\right)+\frac{1}{2}\left(\frac{\zeta}{h / 2}\right)^{3}\right]\right\},
\end{aligned}
$$

where the functions $S$ and $T$ are as yet undetermined; $q^{+}$and $q^{-}, p_{1}^{+}$and $p_{1}^{-}$, and $p_{2}^{+}$and $p_{2}^{-}$are, respectively, the values $\sigma_{\xi}, \tau_{1 \zeta}$, and $\tau_{2 \zeta}$ at the top and bottom surfaces of the shell $(\zeta= \pm h / 2) ; H^{+}, H^{-}$, etc. denote

$$
\begin{aligned}
& H^{+}=1+\frac{h}{2}\left(\frac{1}{R_{1}}+\frac{1}{R_{2}}\right)+\frac{h^{2}}{4} \frac{1}{R_{1} R_{2}}, \\
& H^{-}=1-\frac{h}{2}\left(\frac{1}{R_{1}}+\frac{1}{R_{2}}\right)+\frac{h^{2}}{4} \frac{1}{R_{1} R_{2}}, \\
& H_{1}^{+}=1+\frac{h}{2 R_{2}}, \quad H_{1}^{-}=1-\frac{h}{2 R_{2}}, \\
& H_{2}^{+}=1+\frac{h}{2 R_{1}}, \quad H_{2}^{-}=1-\frac{h}{2 R_{1}},
\end{aligned}
$$

and the coefficient $c$ is introduced on the right-hand side of $\sigma_{\zeta}$ for the purpose of distinguishing between the contributions of transverse shear deformation and normal stress; otherwise, it should be regarded as unity.

It may be mentioned that the inclusion of $w^{\prime}$ and $w^{\prime \prime}$ in $W$ is closely associated with 
the functions $S$ and $T$, respectively. In fact, as will be seen later, if in $\sigma_{\zeta}, S$ and $T$ are set equal to zero, this will be consistent with approximating $W \simeq w$.

By (2.5) and with the aid of $\tau_{12}$ given in (2.9), the expressions for $N_{21}$ and $M_{21}$ may be written as

$$
\left\{\begin{array}{l}
N_{21} \\
M_{21}
\end{array}\right\}=\int_{-h / 2}^{+h / 2}\left[\frac{N_{12}}{h}+\frac{M_{12}}{h^{2} / 6} \frac{\zeta}{h / 2}\right]\left(1+\frac{\zeta}{R_{2}}\right)^{-1}\left\{\begin{array}{l}
1 \\
\zeta
\end{array}\right\}\left(1+\frac{\zeta}{R_{1}}\right) d \zeta
$$

and the truth of the identity

$$
N_{12}+\frac{M_{12}}{R_{1}}=N_{21}+\frac{M_{21}}{R_{2}}
$$

can be directly verified from the expressions for the stress resultants and the stress couples in (2.5).

Since $h / R$ is assumed to be small in comparison with unity, in what follows only terms up to and including $\zeta^{2}$ will be retained in the expansion of $(1+\zeta / R)^{n}$. In particular, use will be made of the approximate expressions of the type

$$
\begin{gathered}
\int_{-h / 2}^{+h / 2}\left(1+\frac{\zeta}{R_{2}}\right)\left(1+\frac{\zeta}{R_{1}}\right)^{-1} d \zeta \simeq h\left[1+\frac{h^{2}}{12 R_{1}}\left(\frac{1}{R_{1}}-\frac{1}{R_{2}}\right)\right], \\
\int_{-h / 2}^{+h / 2}\left(1+\frac{\zeta}{R_{2}}\right)\left(1+\frac{\zeta}{R_{1}}\right)^{-1} \zeta^{2} d \zeta \simeq \frac{h^{3}}{12}\left[1+\frac{3}{20} \frac{h^{2}}{R_{1}}\left(\frac{1}{R_{1}}-\frac{1}{R_{2}}\right)\right] .
\end{gathered}
$$

3. Derivation of stress strain relations. In order to derive an approximate system of stress strain relations consistent with (2.7) and (2.9), use will be made of a variational theorem due to E. Reissner [4]. Although we seek only the stress strain relations, the Euler equations of the variational theorem yield the stress differential equations of equilibrium as well as the required stress strain relations.

The variational equation may be written as

$$
\begin{aligned}
& \delta\left\{\int \int _ { \sigma } \left[\sigma_{1} \epsilon_{1}+\right.\right.\left.\sigma_{2} \epsilon_{2}+\sigma_{\zeta} \epsilon_{\zeta}+\tau_{12} \gamma_{12}+\tau_{1 \zeta} \gamma_{1 \zeta}+\tau_{2 \zeta} \gamma_{2 \zeta}-\Lambda\right] \\
& \cdot\left(1+\frac{\zeta}{R_{1}}\right)\left(1+\frac{\zeta}{R_{2}}\right) \alpha_{1} \alpha_{2} d \xi_{1} d \xi_{2} d \zeta \\
&-\iint_{s_{1}}\left[\left(p_{1}^{+} U_{1}^{+}+p_{2}^{+} U_{2}^{+}+q^{+} W^{+}\right)\left(1+\frac{h}{2 R_{1}}\right)\left(1+\frac{h}{2 R_{2}}\right)\right. \\
&\left.+\left(p_{1}^{-} U_{1}^{-}+p_{2}^{-} U_{2}^{-}+q^{-} W^{-}\right)\left(1-\frac{h}{2 R_{1}}\right)\left(1-\frac{h}{2 R_{2}}\right)\right] \alpha_{1} \alpha_{2} d \xi_{1} d \xi_{2} \\
&\left.-\oint\left[\int_{-h / 2}^{+h / 2}\left(\sigma_{n} U_{n}+\tau_{n t} U_{t}+\tau_{n \zeta} W\right)\left(1+\frac{\zeta}{R_{t}}\right) d \zeta\right] \alpha_{t} d \xi_{t}\right\}=0
\end{aligned}
$$

where

$$
\Lambda=\frac{1}{2 E}\left[\sigma_{1}^{2}+\sigma_{2}^{2}+\sigma_{\zeta}^{2}-2 \nu\left(\sigma_{1} \sigma_{2}+\sigma_{1} \sigma_{\zeta}+\sigma_{2} \sigma_{\zeta}\right)+2(1+\nu)\left(\tau_{12}^{2}+\tau_{1 \zeta}^{2}+\tau_{2 \zeta}^{2}\right)\right] .
$$

$E$ is Young's modulus, $\nu$ is Poisson's ratio, $v$ denotes the volume, $s_{1}$ indicates that part 
of the surface where the surface loads $p_{1}^{+}, p_{2}^{+}, q^{+}$, etc. are prescribed, and $U_{1}^{+}, U_{1}^{-}$, etc. designate $U_{1}\left(\xi_{1}, \xi_{2}, h / 2\right)$ and $U_{1}\left(\xi_{1}, \xi_{2},-h / 2\right)$ respectively.

In the last integral in (3.1a) which represents the energy associated with the edge stresses, the subscripts $n$ and $t$ refer to the normal and tangential directions on the boundary faces. The stress resultants and the stress couples $N_{n}, N_{n t}, V_{n}, M_{n}$, and $M_{n t}$ due to the edge stresses, are defined similar to those in (2.5); in addition, we note that $Q_{n}=$ $\int_{-h / 2}^{+h / 2} \tau_{n \zeta}\left(1+\zeta / R_{t}\right) \zeta^{2} d \zeta$.

Substituting (2.8) and (2.9) into (3.1), the variational equation becomes

$$
\begin{aligned}
& \delta \iiint\left\{\left(\frac{N_{1}}{h}+\frac{M_{1}}{h^{2} / 6} \frac{\zeta}{h / 2}\right)\left(\epsilon_{1}^{0}+\zeta \kappa_{1}^{*}+\frac{1}{2} \zeta^{2} \frac{w^{\prime \prime}}{R_{1}}\right)+\cdots\right. \\
& +c\left[\left(\frac{3}{2} \frac{S}{h}+\frac{T}{4 h} \frac{\zeta}{h / 2}\right)\left(1-\left(\frac{\zeta}{h / 2}\right)^{2}\right)+\frac{1}{2} q^{+} H^{+}\left(1+\frac{3}{2}\left(\frac{\zeta}{h / 2}\right)-\frac{1}{2}\left(\frac{\zeta}{h / 2}\right)^{3}\right)\right. \\
& \left.+\frac{1}{2} q^{-} H^{-}\left(1-\frac{3}{2}\left(\frac{\zeta}{h / 2}\right)+\frac{1}{2}\left(\frac{\zeta}{h / 2}\right)^{3}\right)\right]\left(w^{\prime}+\zeta w^{\prime \prime}\right) \\
& +\left(\frac{N_{12}}{h}+\frac{M_{12}}{h^{2} / 6} \frac{\zeta}{h / 2}\right)\left(\gamma_{1}^{0}+\zeta \delta_{1}\right)+\left(\frac{N_{12}}{h}+\frac{M_{12}}{h^{2} / 6} \frac{\zeta}{h / 2}\right)\left(\frac{1+\zeta / R_{1}}{1+\zeta / R_{2}}\right)\left(\gamma_{2}^{0}+\zeta \delta_{2}\right) \\
& +\left[\frac{3}{2} \frac{V_{1}}{h}\left(1-\left(\frac{\zeta}{h / 2}\right)^{2}\right)-\frac{1}{4}\left\{p_{1}^{+} H_{1}^{+}\left(1-2\left(\frac{\zeta}{h / 2}\right)-3\left(\frac{\zeta}{h / 2}\right)^{2}\right)+\cdots\right\}\right] \\
& \cdot\left(\gamma_{1 \zeta}^{0}+\frac{\zeta}{\alpha_{1}} \frac{\partial w^{\prime}}{\partial \xi_{1}}+\frac{\zeta^{2}}{2 \alpha_{1}} \frac{\partial w^{\prime \prime}}{\partial \xi_{1}}\right)+\cdots-\frac{1}{2 E}\left\{\left(\frac{N_{1}}{h}+\frac{M_{1}}{h^{2} / 6} \frac{\zeta}{h / 2}\right)^{2}\left(\frac{1+\zeta / R_{1}}{1+\zeta / R_{2}}\right)\right. \\
& +\cdots-2 \nu\left[\left(\frac{N_{1}}{h}+\frac{M_{1}}{h^{2} / 6} \frac{\zeta}{h / 2}\right)\left(\frac{N_{2}}{h}+\frac{M_{2}}{h^{2} / 6} \frac{\zeta}{h / 2}\right)+\cdots\right] \\
& \left.+2(1+\nu)\left[\left(\frac{N_{12}}{h}+\frac{M_{12}}{h^{2} / 6} \frac{\zeta}{h / 2}\right)^{2}\left(\frac{1+\zeta / R_{1}}{1+\zeta / R_{2}}\right)+\cdots\right]\right\}\left\{\alpha_{1} \alpha_{2} d \xi_{1} d \xi_{2} d \zeta\right. \\
& -\delta \iint\left\{\left(p_{1} u_{1}+m_{1} \beta_{1}\right)+\left(p_{2} u_{2}+m_{2} \beta_{2}\right)+\left[\left(q^{+} H^{+}-q^{-} H^{-}\right) w\right.\right. \\
& \left.\left.+\frac{h}{2}\left(q^{+} H^{+}+q^{-} H^{-}\right) w^{\prime}+\frac{h^{2}}{8}\left(q^{+} H^{+}-q^{-} H^{-}\right) w^{\prime \prime}\right]\right\} \alpha_{1} \alpha_{2} d \xi_{1} d \xi_{2} \\
& -\oint\left\{N_{n}^{*} u_{n}+M_{n}^{*} \beta_{n}+N_{n}^{*} u_{t}+M_{n}^{*} \beta_{t}+V_{n}^{*} w\right. \\
& \left.+Q_{n}^{*} w^{\prime \prime}+\frac{h^{2}}{12} p_{n} w^{\prime}+\frac{h^{2}}{60} m_{n} w^{\prime \prime}\right\} \alpha_{t} d \xi_{t}=0
\end{aligned}
$$

where

$$
\begin{aligned}
p_{n} & =p_{n}^{+} H_{n}^{+}-p_{n}^{-} H_{n}^{-}, \\
m_{n} & =\frac{h}{2}\left[p_{n}^{+} H_{n}^{+}+p_{n}^{-} H_{n}^{-}\right],
\end{aligned}
$$


the line integral is taken along the coordinate curves which form the boundary of the middle surface, and the starred quantities are the edge resultants.

Integrating (3.2) with respect to $\zeta$, and observing that

$$
\begin{aligned}
\iiint\left(\frac{N_{12}}{h}+\frac{M_{12}}{h^{2} / 6} \frac{\zeta}{h / 2}\right)\left(\gamma_{2}^{0}+\zeta \delta_{2}\right)\left(1+\frac{\zeta}{R_{1}}\right)\left(1+\frac{\zeta}{R_{2}}\right)^{-1} \alpha_{1} \alpha_{2} d \xi_{1} d \xi_{2} d \zeta & \\
& =\iint\left(N_{21} \gamma_{2}^{0}+M_{21} \delta_{2}\right) \alpha_{1} \alpha_{2} d \xi_{1} d \xi_{2},
\end{aligned}
$$

then carrying out the variations using (2.2), (2.11), (2.12) and (2.13) whenever necessary, integrating by parts, and making use of appropriate transformation relations, the following equation is obtained:

$$
\begin{aligned}
\iint & \left\{-\delta u_{1}\left[\frac{\partial\left(\alpha_{2} N_{1}\right)}{\partial \xi_{1}}+\frac{\partial\left(\alpha_{1} N_{21}\right)}{\partial \xi_{2}}+N_{12} \frac{\partial \alpha_{1}}{\partial \xi_{2}}-N_{2} \frac{\partial \alpha_{2}}{\partial \xi_{1}}+\alpha_{1} \alpha_{2}\left(\frac{V_{1}}{R_{1}}+p_{1}\right)\right]\right. \\
& -\delta u_{2}\left[\frac{\partial\left(\alpha_{2} N_{12}\right)}{\partial \xi_{1}}+\frac{\partial\left(\alpha_{1} N_{2}\right)}{\partial \xi_{2}}+N_{21} \frac{\partial \alpha_{2}}{\partial \xi_{1}}-N_{1} \frac{\partial \alpha_{1}}{\partial \xi_{2}}+\alpha_{1} \alpha_{2}\left(\frac{V_{2}}{R_{2}}+p_{2}\right)\right] \\
& -\delta w\left[\frac{\partial\left(\alpha_{2} V_{1}\right)}{\partial \xi_{1}}+\frac{\partial\left(\alpha_{1} V_{2}\right)}{\partial \xi_{2}}-\alpha_{1} \alpha_{2}\left(\frac{N_{1}}{R_{1}}+\frac{N_{2}}{R_{2}}\right)+\alpha_{1} \alpha_{2}\left(q^{+} H^{+}-q^{-} H^{-}\right)\right] \\
& -\delta \beta_{1}\left[\frac{\partial\left(\alpha_{2} M_{1}\right)}{\partial \xi_{1}}+\frac{\partial\left(\alpha_{1} M_{21}\right)}{\partial \xi_{2}}+M_{12} \frac{\partial \alpha_{1}}{\partial \xi_{2}}-M_{2} \frac{\partial \alpha_{2}}{\partial \xi_{1}}-\alpha_{1} \alpha_{2}\left(V_{1}-m_{1}\right)\right] \\
& \left.-\delta \beta_{2}\left[\frac{\partial\left(\alpha_{2} M_{12}\right)}{\partial \xi_{1}}+\frac{\partial\left(\alpha_{1} M_{2}\right)}{\partial \xi_{2}}+M_{21} \frac{\partial \alpha_{2}}{\partial \xi_{1}}-M_{1} \frac{\partial \alpha_{1}}{\partial \xi_{2}}-\alpha_{1} \alpha_{2}\left(V_{2}-m_{2}\right)\right]\right\} d \xi_{1} d \xi_{2} \\
& +\iint\left\{+\delta N_{1}\left[\epsilon_{1}^{0}+\frac{h^{2}}{24} \frac{w^{\prime \prime}}{R_{1}}-\frac{1}{E h}\left\{N_{1}\left(1-\frac{h^{2}}{12 R_{2}}\left(\frac{1}{R_{1}}-\frac{1}{R_{2}}\right)\right)-\nu N_{2}\right.\right.\right. \\
& +M_{1}\left(\frac{1}{R_{1}}-\frac{1}{R_{2}}\right)-\nu c\left(S\left(1+\frac{h^{2}}{20 R_{2}^{2}}\right)-\frac{h}{60 R_{2}} T\right. \\
& \left.\left.\left.+\frac{h}{2} q^{+} H^{+}\left(1-\frac{h}{5 R_{2}}+\frac{h^{2}}{12 R_{2}^{2}}\right)+\frac{h}{2} q^{-} H^{-}\left(1+\frac{h}{5 R_{2}}+\frac{h^{2}}{12 R_{2}^{2}}\right)\right)\right\}\right] \\
& +\delta N_{2}\left[\epsilon_{2}^{0}+\frac{h^{2}}{24} \frac{w^{\prime \prime}}{R_{2}}-\frac{1}{E h}\{\cdots\}\right] \\
& +N_{1}\left(\frac{1}{R_{1}}-\frac{1}{R_{2}}\right)-\frac{6 \nu}{h} c\left(\frac{T}{4}\left(\frac{2}{15}+\frac{h^{2}}{70 R_{2}^{2}}\right)-\frac{h}{10 R_{2}} S\right. \\
& +\delta N_{12}\left[\gamma_{1}^{0}+\gamma_{2}^{0}\left(1-\frac{h^{2}}{12 R_{2}}\left(\frac{1}{R_{1}}-\frac{1}{R_{2}}\right)\right)+\delta_{2} \frac{h^{2}}{12}\left(\frac{1}{R_{1}}-\frac{1}{R_{2}}\right)\right. \\
& \left.+\frac{2(1+\nu)}{E h}\left\{N_{12}\left(1-\frac{h^{2}}{12 R_{2}}\left(\frac{1}{R_{1}}-\frac{1}{R_{2}}\right)\right)+M_{12}\left(\frac{1}{R_{1}}-\frac{1}{R_{2}}\right)\right\}\right] \\
& +\delta M_{1}\left[\kappa_{1}+\frac{w^{\prime}}{R_{1}}-\frac{1}{E h}\left\{\frac{M_{1}}{h^{2} / 12}\left(1-\frac{3}{R_{2}}\left(\frac{1}{R_{1}}-\frac{1}{R_{2}}\right)\right)-12\right.\right. \\
& =M_{2} \\
& \\
&
\end{aligned}
$$




$$
\begin{aligned}
& \left.\left.\left.+\frac{h}{2} q^{+} H^{+}\left(\frac{2}{5}-\frac{h}{6 R_{2}}+\frac{2}{35} \frac{h^{2}}{R_{2}^{2}}\right)-\frac{h}{2} q^{-} H^{-}\left(\frac{2}{5}+\frac{h}{6 R_{2}}+\frac{2}{35} \frac{h^{2}}{R_{2}^{2}}\right)\right)\right\}\right] \\
& +\delta M_{2}\left[\kappa_{2}+\frac{w^{\prime}}{R_{2}}-\frac{1}{E h}\{\cdots\}\right] \\
& +\delta M_{12}\left[\delta_{1}+\delta_{2}\left(1-\frac{3}{20} \frac{h^{2}}{R_{2}}\left(\frac{1}{R_{1}}-\frac{1}{R_{2}}\right)\right)+\gamma_{2}^{0}\left(\frac{1}{R_{1}}-\frac{1}{R_{2}}\right)\right. \\
& \left.-\frac{2(1+\nu)}{E h}\left\{\frac{M_{12}}{h^{2} / 12}\left(1-\frac{3}{20} \frac{h^{2}}{R_{2}}\left(\frac{1}{R_{1}}-\frac{1}{R_{2}}\right)\right)+N_{12}\left(\frac{1}{R_{1}}-\frac{1}{R_{2}}\right)\right\}\right] \\
& +\partial V_{1}\left[\gamma_{15}^{0}+\frac{h^{2}}{40} \frac{1}{\alpha_{1}} \frac{\partial w^{\prime \prime}}{\partial \xi_{1}}-\frac{9}{2} \frac{1+\nu}{E h} V_{1}\left\{\frac{8}{15}-\frac{2 h^{2}}{105 R_{2}}\left(\frac{1}{R_{1}}-\frac{1}{R_{2}}\right)\right\}\right. \\
& \left.+\frac{1+\nu}{5 E}\left\{\frac{m_{1}}{h / 2}\left(1-\frac{h^{2}}{28 R_{2}}\left(\frac{1}{R_{2}}-\frac{1}{R_{1}}\right)\right)+\frac{h}{2} p_{1}\left(\frac{1}{R_{2}}-\frac{1}{R_{1}}\right)\right\}\right] \\
& +\delta V_{2}\left[\gamma_{2 \zeta}^{0}+\frac{h^{2}}{40} \frac{1}{\alpha_{2}} \frac{\partial w^{\prime \prime}}{\partial \xi_{2}}-\frac{9}{2} \frac{1+\nu}{E h} V_{2}\{\cdots\}+\frac{1+\nu}{5 E}\{\cdots\}\right] \\
& +\delta w^{\prime}\left[\left(\frac{M_{1}}{R_{1}}+\frac{M_{2}}{R_{2}}\right)+c\left\{S+\frac{h}{2}\left(q^{+} H^{+}+q^{-} H^{-}\right)\right\}\right. \\
& \left.-\frac{h}{2}\left(q^{+} H^{+}+q^{-} H^{-}\right)-\frac{h^{2}}{12}\left\{\frac{\partial\left(\alpha_{2} p_{1}\right)}{\partial \xi_{1}}+\frac{\partial\left(\alpha_{1} p_{2}\right)}{\partial \xi_{2}}\right\}\right] \\
& +\delta w^{\prime \prime}\left[\frac{h^{2}}{24}\left(\frac{N_{1}}{R_{1}}+\frac{N_{2}}{R_{2}}\right)+c\left\{\frac{h}{60} T+\frac{h^{2}}{10}\left(q^{+} H^{+}-q^{-} H^{-}\right)\right\}\right. \\
& \left.-\frac{h^{2}}{8}\left(q^{+} H^{+}-q^{-} H^{-}\right)-\frac{h^{2}}{60}\left\{\frac{\partial\left(\alpha_{2} m_{1}\right)}{\partial \xi_{1}}+\frac{\partial\left(\alpha_{1} m_{2}\right)}{\partial \xi_{2}}\right\}\right] \\
& +\delta S\left[c w^{\prime}-\frac{1}{E h}\left\{\frac{9}{4} c^{2} S\left(\frac{8}{15}+\frac{2 h}{105}\left(\frac{1}{R_{1}^{2}}+\frac{1}{R_{1} R_{2}}+\frac{1}{R_{2}^{2}}\right)\right)-c^{2} \frac{h}{70} T\left(\frac{1}{R_{1}}+\frac{1}{R_{2}}\right)\right.\right. \\
& +c^{2} \frac{h}{2} q_{+} H^{+}\left(1-\frac{9 h}{70}\left(\frac{1}{R_{1}}+\frac{1}{R_{2}}\right)+\frac{h^{2}}{20}\left(\frac{1}{R_{1}^{2}}+\frac{1}{R_{1} R_{2}}+\frac{1}{R_{2}^{2}}\right)\right) \\
& +c^{2} \frac{h}{2} q_{-} H^{-}\left(1+\frac{9 h}{70}\left(\frac{1}{R_{1}}+\frac{1}{R_{2}}\right)+\frac{h^{2}}{20}\left(\frac{1}{R_{1}^{2}}+\frac{1}{R_{1} R_{2}}+\frac{1}{R_{2}^{2}}\right)\right) \\
& \left.\left.+\nu c\left(\frac{h^{2}}{10 R_{2}} \frac{M_{1}}{h^{2} / 6}+\frac{h^{2}}{10 R_{1}} \frac{M_{2}}{h^{2} / 6}-N_{2}\left(1+\frac{h^{2}}{20 R_{1}^{2}}\right)-N_{1}\left(1+\frac{h^{2}}{20 R_{2}^{2}}\right)\right)\right\}\right] \\
& +\delta T\left[c \frac{h}{60} w^{\prime \prime}-\frac{1}{E h}\left\{-c^{2} \frac{h}{70} S\left(\frac{1}{R_{1}}+\frac{1}{R_{2}}\right)\right.\right. \\
& +c^{2} \frac{T}{16}\left(\frac{8}{105}+\frac{2 h^{2}}{315}\left(\frac{1}{R_{1}^{2}}+\frac{1}{R_{1} R_{2}}+\frac{1}{R_{2}^{2}}\right)\right) \\
& +c^{2} \frac{h}{8} q_{+} H^{+}\left(\frac{6}{35}-\frac{h}{15}\left(\frac{1}{R_{1}}+\frac{1}{R_{2}}\right)+\frac{11 h^{2}}{630}\left(\frac{1}{R_{1}^{2}}+\frac{1}{R_{1} R_{2}}+\frac{1}{R_{2}^{2}}\right)\right)
\end{aligned}
$$




$$
\begin{aligned}
& +c^{2} \frac{h}{8} q_{-} H^{-}\left(\frac{6}{35}+\frac{h}{15}\left(\frac{1}{R_{1}}+\frac{1}{R_{2}}\right)+\frac{11 h^{2}}{630}\left(\frac{1}{R_{1}^{2}}+\frac{1}{R_{1} R_{2}}+\frac{1}{R_{2}^{2}}\right)\right) \\
& -\nu c\left(\frac{h}{60 R_{2}} N_{1}+\frac{h}{60 R_{1}} N_{2}-\frac{h}{4}\left(\frac{2}{15}+\frac{h^{2}}{70 R_{2}^{2}}\right) \frac{M_{1}}{h^{2} / 6}\right. \\
& \left.\left.\left.\left.-\frac{h}{4}\left(\frac{2}{15}+\frac{h^{2}}{70 R_{1}^{2}}\right) \frac{M_{2}}{h^{2} / 6}\right)\right\}\right]\right\} \alpha_{1} \alpha_{2} d \xi_{1} d \xi_{2} \\
& -\oint\left\{\left(N_{n}^{*}-N_{n}\right) \delta u_{n}+\left(N_{n t}^{*}-N_{n t}\right) \delta u_{t}+\left(M_{n}^{*}-M_{n}\right) \delta \beta_{n}\right. \\
& \left.+\left(M_{n t}^{*}-M_{n \imath}\right) \delta \beta_{\imath}+\left(V_{n}^{*}-V_{n}\right) \delta w+\frac{h^{2}}{40}\left(V_{n}^{*}-V_{n}\right) \delta w^{\prime \prime}\right\} \alpha_{\iota} d \xi_{t}=0,
\end{aligned}
$$

where, in deducing the coefficients of $\delta N_{12}$ and $\delta M_{12}$, use has been made of an explicit form of (2.11).

As the contents of every bracket of the surface integral in (3.4) must vanish, the first five of the resulting equations are the stress differential equations of equilibrium for a thin shell. The coefficients of $\delta w^{\prime}$ and $\delta w^{\prime \prime}$ are also contributions to equilibrium, while the remaining ten equations are the approximate stress strain relations containing the effects of both transverse normal stress and shear deformation, as well as appropriate expressions for the functions $S$ and $T$. These functions, together with $w^{\prime}$ and $w^{\prime \prime}$, given by the last four brackets in the surface integral, when simplified read:

$$
\begin{gathered}
w^{\prime}=-\frac{\nu}{E h}\left(N_{1}+N_{2}\right)+\frac{c}{E h}\left[\frac{6}{5} S+\frac{h}{2}\left(q^{+} H^{+}+q^{-} H^{-}\right)\right], \\
w^{\prime \prime}=-\frac{\nu}{E h} \frac{M_{1}+M_{2}}{h^{2} / 12}+\frac{c}{E h}\left[\frac{2}{7} \frac{T}{h}+\frac{9}{7}\left(q^{+} H^{+}-q^{-} H^{-}\right)\right], \\
S=-\left(\frac{M_{1}}{R_{1}}+\frac{M_{2}}{R_{2}}\right)+\frac{h^{2}}{12 \alpha_{1} \alpha_{2}}\left[\frac{\partial}{\partial \xi_{1}}\left(\alpha_{2} p_{1}\right)+\frac{\partial}{\partial \xi_{2}}\left(\alpha_{1} p_{2}\right)\right], \\
\frac{T}{h}=-\left(\frac{N_{1}}{R_{1}}+\frac{N_{2}}{R_{2}}\right)+\frac{1}{\alpha_{1} \alpha_{2}}\left[\frac{\partial}{\partial \xi_{1}}\left(\alpha_{2} m_{1}\right)+\frac{\partial}{\partial \xi_{2}}\left(\alpha_{1} m_{2}\right)\right],
\end{gathered}
$$

which reduce to those given previously by E. Reissner for axisymmetric shells of revolution. Equations $\left(20^{\prime}\right)$ and (21) of Ref. [3] should read, respectively,

$$
\frac{T}{h}=-\frac{1}{2}\left(\frac{N_{\xi}}{R_{\xi}}+\frac{N_{\theta}}{R_{\theta}}\right) \text { and } \omega_{M}=\frac{1}{E_{\zeta} h}\left[\frac{2}{7} \frac{T}{h}-12 \nu_{\zeta} \frac{M_{\xi}+M_{\theta}}{h^{2}}\right] .
$$

In this connection, compare the expressions for $\sigma_{5}$ given by (2.9) of the present paper and (18) of Ref. [3].

It is evident that the independent vanishing of each term of the line integral in (3.4) furnishes the required boundary conditions along each edge of the shell; these are either the stress or displacement boundary conditions. For example, we have for the first term of the line integral either $u_{n}=U_{n}\left(\xi_{n}, \xi_{t}, 0\right)$ prescribed, or $N_{n}=N_{n}^{*}$, both along $\xi_{n}=$ constant. It is noteworthy that, in view of the assumed form of the dis- 
placements (2.7) and the stresses (2.9), the derived boundary conditions represent some simplification compared with those given in Ref. [1].

If the effect of normal stress is neglected and only that of transverse shear deformation is retained, the approximate stress strain relations simplify somewhat. This is achieved by putting equal to zero the coefficient $c$ in the stress strain relations and is consistent with the approximation $W=w$ in (2.7). Neglecting second order corrections in $h / R$ in comparison with first order corrections, then, following a lengthy algebraic manipulation and using Cramer's rule, the resulting stress strain relations which satisfy the identity (2.12) become

$$
\begin{aligned}
& N_{1}=\frac{E h}{1-\nu^{2}}\left[\left(\epsilon_{1}^{0}+\nu \epsilon_{2}^{0}\right)-\frac{h^{2}}{12}\left(\frac{1}{R_{1}}-\frac{1}{R_{2}}\right) \kappa_{1}\right], \\
& N_{2}=\frac{E h}{1-\nu^{2}}\left[\left(\epsilon_{2}^{0}+\nu \epsilon_{1}^{0}\right)-\frac{h^{2}}{12}\left(\frac{1}{R_{2}}-\frac{1}{R_{1}}\right) \kappa_{2}\right], \\
& N_{12}=G h\left[\left(\gamma_{1}^{0}+\gamma_{2}^{0}\right)+\frac{h^{2}}{12}\left(\frac{1}{R_{1}}-\frac{1}{R_{2}}\right)\left(\frac{\gamma_{1}^{0}}{R_{1}}-\delta_{1}\right)\right], \\
& N_{21}=G h\left[\left(\gamma_{1}^{0}+\gamma_{2}^{0}\right)+\frac{h^{2}}{12}\left(\frac{1}{R_{2}}-\frac{1}{R_{1}}\right)\left(\frac{\gamma_{2}^{0}}{R_{2}}-\delta_{2}\right)\right], \\
& M_{1}=D\left[\left(\kappa_{1}+\nu \kappa_{2}\right)-\left(\frac{1}{R_{1}}-\frac{1}{R_{2}}\right) \epsilon_{1}^{0}\right], \\
& M_{2}=D\left[\left(\kappa_{2}+\nu \kappa_{1}\right)-\left(\frac{1}{R_{2}}-\frac{1}{R_{1}}\right) \epsilon_{2}^{0}\right], \\
& M_{12}=\frac{1-\nu}{2} D\left[\left(\delta_{1}+\delta_{2}\right)+\left(\frac{1}{R_{2}}-\frac{1}{R_{1}}\right) \gamma_{1}^{0}\right], \\
& M_{21}=\frac{1-\nu}{2} D\left[\left(\delta_{1}+\delta_{2}\right)+\left(\frac{1}{R_{1}}-\frac{1}{R_{2}}\right) \gamma_{2}^{0}\right], \\
& V_{1}=\frac{5}{6} G h\left[\frac{1}{\alpha_{1}} \frac{\partial w}{\partial \xi_{1}}-\left(\frac{u_{1}}{R_{1}}-\beta_{1}\right)\right]+\frac{1}{6}\left[m_{1}+\frac{h^{2}}{4} p_{1}\left(\frac{1}{R_{2}}-\frac{1}{R_{1}}\right)\right], \\
& V_{2}=\frac{5}{6} G h\left[\frac{1}{\alpha_{2}} \frac{\partial w}{\partial \xi_{2}}-\left(\frac{u_{2}}{R_{2}}-\beta_{2}\right)\right]+\frac{1}{6}\left[m_{2}+\frac{h^{2}}{4} p_{2}\left(\frac{1}{R_{1}}-\frac{1}{R_{2}}\right)\right]
\end{aligned}
$$

where $G$ is shear modulus and $D=E h^{3} / 12\left(1-\nu^{2}\right)$

The effect of normal stress, if included in the stress strain relations, will result in the following expressions for $N_{1}, M_{1}$, and $V_{1}$ :

$$
\begin{aligned}
N_{1} & =\frac{E h}{1-\nu^{2}}\left[\left(\epsilon_{1}^{0}+\nu \epsilon_{2}^{0}\right)-\frac{h^{2}}{12}\left(\frac{1}{R_{1}}-\frac{1}{R_{2}}\right) \kappa_{1}\right] \\
& +\left\{-\frac{\nu}{1-\nu} \frac{h^{2}}{12}\left[\frac{1}{2}\left(\frac{1}{R_{1}}+\frac{\nu}{R_{2}}\right)\left(\kappa_{1}+\kappa_{2}\right)+\frac{\kappa_{1}+\nu \kappa_{2}}{R_{1}}+\frac{\kappa_{2}+\nu \kappa_{1}}{R_{2}}\right]\right. \\
& \left.+\frac{\nu}{1-\nu} \frac{h}{2}\left(q^{+}+q^{-}\right)\right\},
\end{aligned}
$$




$$
\begin{aligned}
M_{1} & =D\left[\left(\kappa_{1}+\nu \kappa_{2}\right)-\left(\frac{1}{R_{1}}-\frac{1}{R_{2}}\right) \epsilon_{1}^{0}\right] \\
& +\left\{-\frac{\nu}{1-\nu}\left[\left(\frac{1}{R_{1}}+\frac{\nu}{R_{2}}\right)\left(\epsilon_{1}^{0}+\epsilon_{2}^{0}\right)+\frac{1}{5}\left(\frac{\epsilon_{1}^{0}+\nu \epsilon_{2}^{0}}{R_{1}}+\frac{\epsilon_{2}^{0}+\nu \epsilon_{1}^{0}}{R_{2}}\right)\right]\right. \\
& \left.+\frac{6}{5} \frac{\nu}{1-\nu} \frac{h^{2}}{12}\left(q^{+}-q^{-}\right)\right\}, \\
V_{1} & =\frac{5}{6} G h\left[\frac{1}{\alpha_{1}} \frac{\partial w}{\partial \xi_{1}}-\left(\frac{u_{1}}{R_{1}}-\beta_{1}\right)\right]+\frac{1}{6}\left[m_{1}+\frac{h^{2}}{4} p_{1}\left(\frac{1}{R_{2}}-\frac{1}{R_{1}}\right)\right] \\
& +\left\{-\frac{\nu}{1-\nu} \frac{h^{2}}{40} \frac{\partial}{\partial \xi_{1}}\left[(1+\nu)\left(\kappa_{1}+\kappa_{2}\right)-\left(\frac{1}{R_{1}}-\frac{1}{R_{2}}\right)\left(\epsilon_{1}^{0}-\epsilon_{2}^{0}\right)\right.\right. \\
& \left.-\frac{\nu}{1-\nu}\left((1+\nu)\left(\frac{1}{R_{1}}+\frac{1}{R_{2}}\right)\left(\epsilon_{1}^{0}+\epsilon_{2}^{0}\right)+\frac{2}{5} \epsilon_{1}^{0}\left(\frac{1}{R_{1}}+\frac{\nu}{R_{2}}\right)+\frac{2}{5} \epsilon_{2}^{0}\left(\frac{1}{R_{2}}+\frac{\nu}{R_{1}}\right)\right)\right] \\
& \left.-\frac{3 h^{2}}{50} \frac{\nu^{2}}{(1-\nu) E h} \frac{\partial}{\partial \xi_{1}}\left(q^{+}-q^{-}\right)\right\}
\end{aligned}
$$

and analogous expressions for $N_{2}, M_{2}$, and $V_{2}$, where the coefficient $c$ is taken as unity. The expressions for $N_{12}, N_{21}, M_{12}$, and $M_{21}$ remain unaltered, as given by (3.6).

It may be noted that, except for the shear resultants $V_{1}$ and $V_{2}$ and their effects on $\kappa_{1}$ and $\kappa_{2}$, the stress strain relations (3.7) are similar in form to those commonly known as Love's second approximation. Equations (3.6), on the other hand, appear to contain the necessary corrections (due to the effect of transverse shear deformation) to Love's first approximation.

4. Note on the effect of rotatory inertia. In the classical treatment of vibration of elastic shells and plates, in addition to the effect of normal stress and transverse shear deformation, the effect of rotatory inertia is also neglected. To include the latter in the results of the preceding section, the volume integral in (3.1a) should be modified in that the negative of kinetic energy

$$
\frac{1}{2} \rho\left[\left(\frac{\partial U_{1}}{\partial t}\right)^{2}+\left(\frac{\partial U_{2}}{\partial t}\right)^{2}+\left(\frac{\partial W}{\partial t}\right)^{2}\right],
$$

where $\rho$ is the mass density and $t$ denotes time, should be added to the integrand. Consequently, the surface integral in (3.4) is modified as follows:

To each of the five coefficients of $\delta u_{1}, \delta u_{2}, \delta w, \delta \beta_{1}$, and $\delta \beta_{2}$, in the surface integral (3.4), should be added the following expressions, respectively:

$$
\begin{aligned}
\delta u_{1}:-\rho h\left[\left(1+\frac{h^{2}}{12 R_{1} R_{2}}\right) \frac{\partial^{2} u_{1}}{\partial t^{2}}+\frac{h^{2}}{12}\left(\frac{1}{R_{1}}+\frac{1}{R_{2}}\right) \frac{\partial^{2} \beta_{1}}{\partial t^{2}}\right] \alpha_{1} \alpha_{2}, \\
\delta u_{2}:-\rho h\left[\left(1+\frac{h^{2}}{12 R_{1} R_{2}}\right) \frac{\partial^{2} u_{2}}{\partial t^{2}}+\frac{h^{2}}{12}\left(\frac{1}{R_{1}}+\frac{1}{R_{2}}\right) \frac{\partial^{2} \beta_{2}}{\partial t^{2}}\right] \alpha_{1} \alpha_{2}, \\
\delta w:-\rho h\left[\left(1+\frac{h^{2}}{12 R_{1} R_{2}}\right) \frac{\partial^{2} w}{\partial t^{2}}+\frac{h^{2}}{12}\left(\frac{1}{R_{1}}+\frac{1}{R_{2}}\right) \frac{\partial^{2} w^{\prime}}{\partial t^{2}}\right. \\
+ \\
\left.+\frac{h^{2}}{24}\left(1+\frac{3}{20} \frac{h^{2}}{R_{1} R_{2}}\right) \frac{\partial^{2} w^{\prime \prime}}{\partial t^{2}}\right] \alpha_{1} \alpha_{2},
\end{aligned}
$$




$$
\begin{aligned}
& \delta \beta_{1}:-\rho \frac{h^{3}}{12}\left[\left(\frac{1}{R_{1}}+\frac{1}{R_{2}}\right) \frac{\partial^{2} u_{1}}{\partial t^{2}}+\left(1+\frac{3}{20} \frac{h^{2}}{R_{1} R_{2}}\right) \frac{\partial^{2} \beta_{1}}{\partial t^{2}}\right] \alpha_{1} \alpha_{2}, \\
& \delta \beta_{2}:-\rho \frac{h^{3}}{12}\left[\left(\frac{1}{R_{1}}+\frac{1}{R_{2}}\right) \frac{\partial^{2} u_{2}}{\partial t^{2}}+\left(1+\frac{3}{20} \frac{h^{2}}{R_{1} R_{2}}\right) \frac{\partial^{2} \beta_{2}}{\partial t^{2}}\right] \alpha_{1} \alpha_{2} .
\end{aligned}
$$

Also, the coefficients of $\delta w^{\prime}$ and $\delta w^{\prime \prime}$ in the surface integral (3.4) are modified, respectively, by addition of the expressions

$$
\begin{aligned}
& \delta w^{\prime}: \quad+\rho \frac{h^{3}}{12}\left[\left(\frac{1}{R_{1}}+\frac{1}{R_{2}}\right) \frac{\partial^{2} w}{\partial t^{2}}+\left(1+\frac{3}{20} \frac{h^{2}}{R_{1} R_{2}}\right) \frac{\partial^{2} w^{\prime}}{\partial t^{2}}\right. \\
& \left.+\frac{3 h^{2}}{40}\left(\frac{1}{R_{1}}+\frac{1}{R_{2}}\right) \frac{\partial^{2} w^{\prime \prime}}{\partial t^{2}}-\right] . \\
& \delta w^{\prime \prime}: \quad+\rho \frac{h^{3}}{24}\left[\left(1+\frac{3}{20} \frac{h^{2}}{R_{1} R_{2}}\right) \frac{\partial^{2} w}{\partial t^{2}}+\frac{3}{20}\left(\frac{1}{R_{1}}+\frac{1}{R_{2}}\right) \frac{\partial^{2} w^{\prime}}{\partial t^{2}}\right. \\
& \left.+\frac{3 h^{2}}{40}\left(1+\frac{5}{28} \frac{h^{2}}{R_{1} R_{2}}\right) \frac{\partial^{2} w^{\prime \prime}}{\partial t^{2}}\right] \text {. }
\end{aligned}
$$

It is interesting to note that when $W \simeq w$ and for the case of a flat plate where $R_{1}=R_{2}=\infty$, the expressions (4.2) simplify considerably.

\section{REFERENCES}

1. F. B. Hildebrand, E. Reissner, and G. B. Thomas, Notes on the foundations of the theory of small displacements of orthotropic shells, Natl. Advisory Comm. Aeronaut., Tech. Notes No. 1833 (1949)

2. A. E. Green and W. Zerna, The equilibrium of thin elastic shells, Quart. J. Mech. and Appl. Math. 3, 9-22 (1950)

3. Eric Reissner, Stress strain relations in the theory of thin elastic shells, J. Math. Phys. 31, 109-119 (1952)

4. Eric Reissner, On a variational theorem in elasticity, J. Math. Phys. 29, 90-95 (1950)

5. A. E. H. Love, $A$ treatise on the mathematical theory of elasticity, Dover Publications, 4th ed., 1944 